\title{
Senderos
}

\section{Historicidad de procesos de exclusión y discriminación para grupos minoritarios en educación superior.}

\author{
Historicity of processes of exclusion and discrimination \\ for minority groups in higher education.
}

\section{Autores \\ Jorge Ivan Correa Alzate ${ }^{1}$ \\ Recibido: 20 de mayo de 2017. \\ Aprobado: 25 de octubre de 2017.}

\footnotetext{
1 El artículo corresponde a uno de los apartados de la tesis doctoral "La construcción de la justicia curricular en la educación superior, situada en la ecología de saberes de grupos minoritarios discriminados y excluidos". Doctorado en Humanidades de la Universidad Nacional de Rosario Argentina.

Docente titular del Tecnológico de Antioquia. Director del grupo de investigación Senderos. jcorrea@tdea.edu.co
}

\section{Resumen}

Algunos grupos sociales históricamente excluidos se han convertido en el blanco de estigmas, como población afrocolombiana, LGBTI, mujeres, personas privadas de la libertad, niños y niñas, personas en pobreza extrema, indígenas, migrantes, personas en situación de discapacidad, adultos mayores, y otros emergentes como las tribus urbanas. Para ellos y ellas, tradicionalmente excluidos del ejercicio de sus derechos fundamentales, se diseñan políticas públicas para establecer su protección y restitución, esto no es garantía de una vida sin exclusiones o discriminaciones.

Palabras clave: discriminación, derechos fundamentales, exclusión, justicia social, grupo minoritario. 


\begin{abstract}
Some historically excluded social groups have become the target of stigmas, as Afro-Colombian population, LGBTI, women, people deprived of liberty, boys and girls, people in extreme poverty, indigenous people, migrants, people with disabilities, elderly people, and other emerging as the urban tribes. For them, traditionally excluded from the exercise
\end{abstract}

of their fundamental rights, public policies are designed to establish their protection and restitution, this is not a guarantee of a life without exclusions or discriminations.

Key words: discrimination, fundamental rights, exclusion, social justice, minority group.

\section{Desarrollo}

Se reconoce por grupo minoritario aquellos colectivos más vulnerados en sus derechos y a quienes históricamente se les han coartado oportunidades económicas, educativas, en salud y trabajo, que afectan la posibilidad de llevar una vida con dignidad. Correa, considera que además de les ha vulnerado en

...sus identidades, que han desencadenado situaciones de marginalidad y exclusión, y además se les ha coartado el acceso a la educación. En la actualidad son objeto de las políticas de inclusión educativa que promueven oportunidades educativas para el acceso y la participación (2018, p.6).

Como consecuencia, son marginados o excluidos en la sociedad y son discriminados por el no reconocimiento de su identidad, lo que se agudiza en la interacción con otros, producto de los desencuentros e incomprensiones, y afecta la convivencia por intolerancia. El concepto se complementa con los planteamientos de Enrique Dussel, quien afirma que el otro es el pueblo latinoamericano (es la periferia, lo marginado, los empobrecidos). Metafóricamente, en el contexto universitario, los otros son las minorías discriminadas y excluidas, cuya realidad se caracteriza por la desvaloración de sus capacidades. Dussel lo expresa así:

A raíz de la modernidad se crea la humanidad sobrante (desechable, desempleada, excluida) aumenta así la proporción de sujetos necesitados no solventes tanto en el centro como en la periferia en América latina, África o Asia y por lo tanto hoy constatamos cómo la miseria crece en todo el planeta. (1997, p. 65)

Este concepto de grupos excluidos refleja una situación del ser humano, ha tomado nuevas significaciones y tendrá que superarse para realizar nuevas configuraciones en la educación. En palabras de Boaventura Santos:

El campesino africano o latinoamericano puede encontrarse con el ejecutivo del Banco Mundial: es un encuentro simultáneo, pero no contemporáneo, porque el campesino latinoamericano o africano es "residual” y el ejecutivo es "avanzado". Lo importante, entonces, es reconocer que el campesino es a su manera tan contemporáneo como el ejecutivo, y eliminar el concepto de residualidad. (2005, p. 27) 
Da a entender Santos, al reflexionar sobre la realidad actualizada en época y escenarios, que cada grupo en su momento histórico emerge en una situación o en varias situaciones de desigualdad social y su itinerario con la vulneración es particular, indígenas, afrocolombianos, personas con discapacidad, adultos mayores, mujeres y comunidad LGBTI han tenido vivencias y trayectorias específicas, evidenciadas en hechos políticos, sociales y culturales, que explican su exclusión de la educación y que además revelan otra situación como es la discriminación a sus identidades. Un elemento común entre estos grupos es la violación a sus derechos fundamentales, y en el campo educativo han recibido una formación compensatoria y homogeneizadora, con diferentes matices según la época. Hoy la educación, desde las políticas de los estados, propende por la inclusión para estos grupos que históricamente han sido excluidos y discriminados.

Esta injusticia histórica con ellos se ha querido refrendar, y una de las acciones afirmativas que se han impulsado en las últimas décadas corresponde al acceso a la educación, sin embargo, para los estudiantes que ingresan al sistema educativo por alguna situación de vulnerabilidad, el simple acceso no es garantía de permanencia y de culminación exitosa con la graduación; entre las razones se encuentra que la propuesta educativa no responde a sus expectativas en coherencia con sus trayectorias de vida, se enfrentan a currículos homogeneizadores que no articulan sus saberes del contexto, las didácticas y los recursos pedagógicos no son pertinentes a sus formas de aprender o las actitudes de docentes y pares no acogen sus necesidades de participación; adicionalmente, los currículos continúan siendo profesionalizantes e invisibilizan sus saberes y prácticas, y en algunos casos son de tipo compensatorio, y los estudiantes que alcanzan la titulación no logran empoderamiento para salir de sus situaciones de desventaja social. En consecuencia, las desigualdades se acentúan principalmente en el logro del aprendizaje.

Como alternativa a estas circunstancias, en la actualidad se promocionan currículos contrahegemónicos en la educación superior, que se fundamenten en la justicia curricular y en el reconocimiento de los saberes propios de los diferentes grupos minoritarios, para comprender su realidad educativa, vale la pena identificar unos hitos implícitos en la trayectoria de diferentes épocas.

La exclusión de la educación a los grupos reconocidos como minoritarios ha estado relacionada en el ámbito mundial con la negación de sus derechos, amparada en políticas conservadoras o ideologías relacionadas con el rol de las personas en la sociedad. A manera de ejemplo, en el caso de Colombia, Henderson expresa que entre los años 1930-1940:

La sociedad colombiana titubeaba entre la tradición y el cambio. Algunos imaginarios y estereotipos raciales y sexuales permanecían aferrados, como era el caso de la condición sumisa endilgada a las mujeres, creencia motivada en gran medida por la considerable influencia de la religión, ya fuera en su conducta privada o pública. (2006, pp. 415-416) 
El negro en el estigma de esclavo, no fue considerado sujeto de la educación por ser una raza impura en relación con la de las castas y de las monarquías, y por el rol de "servir a su amo", con características de su deber como la obediencia y el trabajar sin protestar; mientras que para la mujer su papel consistía en tener hijos, cuidar al marido y acceder únicamente al aprendizaje de la costura, formación asignada exclusivamente a comunidades religiosas.

A las personas con discapacidad se les asociaba con enfermedad, en consecuencia la atención que recibían era en salud y su cuidado se asignaba a comunidades religiosas con capacidad de abnegación, por lo tanto, son invisibilizados de la educación por considerar que no tenían inteligencia para lograr los aprendizajes; este pensamiento se expande a las comunidades indígenas, habitantes de otro mundo geográfico, "salvajes", que debieron ser evangelizados por no creer en el dios de los católicos.

A lo largo de la historia se encuentra que principalmente estos cuatro grupos, mujeres, indígenas, personas con discapacidad y afrocolombianos, han sido excluidos de la educación con fundamento en posturas irracionales, situación que a través de la práctica se ha legitimado en las instituciones y siempre ha estado en la relación entre política y religión para justificar determinadas acciones hacia el otro. Este hecho lleva a entender que la escuela y la educación históricamente han excluido, y han tenido que reconocer de manera gradual los derechos de algunos grupos en la sociedad, y les han abierto y brindado espacios para el logro de aprendizajes.

En cada grupo el objeto de la exclusión o discriminación ha tenido diferentes fundamentos que cobran sentido según la época y el espacio en el que se originan, lo más reiterativo ha sido la subvaloración de las capacidades. Para el caso de las mujeres, ha sido el primer grupo al que se le reconocen sus capacidades, sin embargo aún persisten estigmas sociales que restringen una apertura participativa plena. En el campo educativo las acciones para restituir los derechos para todos los grupos han sido emergentes y de carácter compensatorio.

La estigmatización que cubre a estos grupos, silencia a individuos y comunidades, amilana sus fortalezas, incrementa sus debilidades, aísla a la gente y la limita en sus derechos. Desde mediados del siglo XX, los organismos internacionales vienen ocupándose del tema a partir de promover políticas y diálogos mundiales y regionales, $\mathrm{y}$ han presentado informes y recomendaciones para combatir cualquier acto discriminatorio. Así lo demuestran algunos estudios que dan cuenta de los trabajos que se llevan a cabo en todos los continentes.

La Unión Europea está organizada en tres instituciones principales para atender los fenómenos sociales de discriminación: el Consejo de Europa, la Comisión Europea, que es a la vez el órgano ejecutivo, y el Parlamento Europeo. A través de estos, se implementan ocho programas especiales relacionados con la no discriminación: Programa de Lucha contra la Discriminación, Programa de Igualdad y No Discriminación en la Europa Ampliada, Año Europeo de Igualdades y Oportunidades 
para Todos, la Estrategia Marco para la No Discriminación y la Igualdad de Oportunidades para Todos, Estrategia de los Derechos de la Infancia, Programa de Acciones Sociales para Grupos Determinados, Programa de Lucha contra el Racismo, la Xenofobia y el Antisemitismo, y Programa de Igualdad de Oportunidades para Hombres y Mujeres (2006, p. 24).

En el caso de África Central, desde 1999 existe el Centro Subregional para los Derechos Humanos y la Democracia. Las condiciones estructurales, políticas, económicas, sociales y culturales dificultan dimensionar los avances del Centro en materia de lucha contra la discriminación, y aunque de manera formal se protege a las mujeres, los niños y los refugiados, se lucha contra la discriminación por motivos raciales y de identidad homosexual y se promueven los principios y valores de la democracia, los hechos se agudizan por las bajas oportunidades educativas y la situación de extrema pobreza.

En Asia los derechos humanos son resultado de la pobreza extendida, la violencia contra mujeres y niños y la ausencia de independencia judicial, así como la protección institucional contra cualquier forma de discriminación es parte inherente a los regímenes democráticos. A modo de ejemplo, aun en los países más avanzados, como la India, los grupos de la diversidad sexual no gozan de derecho alguno, incluso son penalizados con la muerte, y las mujeres son invisibilizadas en la política.

Oceanía, principalmente en Australia y Nueva Zelanda, enfrenta desafíos relacionados con sus poblaciones indígenas, grupos migrantes y otras minorías raciales, pero trabajan de manera eficaz en estas situaciones, de modo que el derecho a la no discriminación es, al menos parcialmente, parte de esta protección.

En América Latina, se reconoce la existencia de normas, agencias y mecanismos de reparación del daño en materia de no discriminación, la cual se agudiza por las condiciones de vida de las personas, culturales, de salud, educación, vivienda y trabajo; no todos los países están provistos de estos mecanismos para garantizar los derechos. La atención institucional a los problemas derivados de la discriminación, o asociados con ella, es heterogénea tanto en cuanto a organismos o legislaciones como a la protección de ciertos grupos sociales. Adicionalmente, la educación es un tema que está en las agendas políticas desde la década de los ochenta por las desigualdades existentes en cobertura y calidad.

En Colombia, la legislación se actualiza mediante las leyes 1482 de 2011 y 1752 de 2015, ambas de carácter penal, que conciben: "actos de discriminación por razones de raza, etnia, religión, nacionalidad, ideología política o filosófica, sexo u orientación sexual, discapacidad y demás”. (Artículo 1). Las prácticas de discriminación se presentan hacia las personas por sus características, la población LGBTI es uno de los grupos sobre los que mayor se acentúan. 
Esta corta ilustración deja ver que la discriminación es una situación mundial que tiene diferentes matices en cada continente, que producto de las relaciones de poder y la existencia de desigualdades sociales, repercute en la vulneración de los derechos de las personas, en la invisibilización como sujetos políticos y en la negación a la identidad, comprendida como la incapacidad que tiene el sujeto de construirse a sí mismo en su interrelación con los demás. Todo ello implica comprender multiplicidad de subjetividades que derivan en la incorporación de diversas opciones de identidades, que son dadas por el mundo externo a los sujetos y se establecen en cuanto a creencias religiosas y culturales, el rol de género, las opciones sexuales, etc. La educación ha negado o visto como problemáticas estas identidades con fundamento en parámetros establecidos por la sociedad y en un estereotipo sobre la capacidad de aprendizaje, por el cual se subvalora la posibilidad de ser educado o se niega una educación pertinente en coherencia con sus necesidades.

A partir de la declaración de los derechos humanos, en el año 1948, se empezaron a reconocer los derechos en igualdad de condiciones para todas las personas. El artículo 2 establece que: "Toda persona tiene todos los derechos y libertades proclamados en esta Declaración, sin distinción alguna de raza, color, sexo, idioma, religión, opinión política o de cualquier otra índole, origen nacional o social, posición económica, nacimiento o cualquier otra condición”. Y para interés de este escrito, el artículo 26:

Toda persona tiene derecho a la educación. La educación debe ser gratuita, al menos en lo concerniente a la instrucción elemental y fundamental. La instrucción elemental será obligatoria. La instrucción técnica y profesional habrá de ser generalizada; el acceso a los estudios superiores será igual para todos, en función de los méritos respectivos.

Tres aspectos de la declaración son importantes de resaltar: 1) el derecho a la educación; 2) que las características de las personas no son causa de la negación de los derechos fundamentales, incluida la educación, y 3) que el acceso a la educación superior está sujeto a capacidades de las personas. Sin embargo, es un hito que marca un desenlace importante en contraposición a la exclusión a la educación y les brinda a las personas fundamentos jurídicos para que reclamen sus derechos.

La promulgación de los derechos humanos no fue a la par con la transformación de los sistemas educativos, y la promoción del acceso a la educación para todas las personas evidenció prácticas culturales discriminatorias fundamentadas, para negar el derecho, en las características de los sujetos, se agudizaron hechos sociales como el racismo, la homofobia, e incluso el feminismo, este último por la influencia europea en nuestro país.

La orientación de la educación siguió igual, a pesar del ingreso al sistema de los sujetos que habían sido históricamente invisibilizados; es evidente que el acceso a la educación para los grupos reconocidos como minoritarios se marca en dos fenómenos, exclusión y discriminación. Las características como color de piel, credo, ideología, cultura 
y preferencia sexual son el eje para continuar con estereotipos, y la educación ha necesitado de mecanismos que protejan el derecho. Solo a partir de 1990 la educación empezó a ser tenida en cuenta en las agendas políticas, reconociendo que el derecho es para todos, sin exclusiones, e involucra reconocer e implementar estrategias para las diferentes formas de aprendizaje y de comunicación de las personas.

Podemos decir que la discriminación se caracteriza por ser una situación culturalmente fundada, con presencia sistemática, con matices diversos y socialmente extendida, a través de la cual se desprecia a una persona o grupo de personas sobre la base de un prejuicio negativo o un estigma relacionado con una desventaja inmerecida, y tiene por efecto (intencional o no) que afecta sus derechos y libertades fundamentales y limita sus oportunidades como sujeto íntegro y político. En estos procesos discriminatorios, el lenguaje ha sido fundamental para señalar a partir de "expresiones desaprobatorias, peyorativas e incorporadas, las cuales provienen desde imaginarios, estereotipos y prejuicios” (Echavarria, Urrea y Zapata, 2015, p.65)

Los estereotipos han evidenciado prácticas discriminatorias profundas por preferencia sexual. El sistema educativo fue dando posibilidad de ingreso a los diferentes grupos excluidos, con algunas resistencias de docentes, familias y sociedades tradicionalistas; surgiendo el rechazo abierto a la educación de estudiantes LGBTI; en el caso colombiano, la Constitución Política les reconoce el derecho a la libre expresión y tuvo que establecer mecanismos para garantizar el acceso y la permanencia en la educación.

En cuanto a la exclusión, Abrahamson reconoce que los individuos son excluidos socialmente cuando:

Sufren desventajas generalizadas en términos de educación, formación profesional, empleo, recursos de financiación de vivienda, etc.; b) sus oportunidades de acceder a las principales instituciones sociales que distribuyen estas oportunidades de vida son sustancialmente inferiores que las del resto de la población; c) estas desventajas persisten en el tiempo. (1997, p. 123)

Ambas, exclusión y discriminación, tienen relación en la multiplicidad de factores causales de tipo social, cultural y político, mostrando la realidad para los grupos minoritarios en un entramado de complejidades, caracterizada en la disminución de sus capacidades, la falta de oportunidades y con la carga negativa a sus identidades; le corresponde a la educación aportar para romper este círculo de circunstancias, de manera que ellos encuentren alternativas para salir de su situación de vulnerabilidad y segregación.

Estas situaciones que viven los grupos minoritarios, Correa y Restrepo plantean:

El entramado social en la que conviven los estudiantes se intersectan por diversos sistemas de exclusión y discriminación, no es solo el hecho de ingresar a la institución si no de permanecer en ella superando barreras de todo tipo, 
estas relacionadas con las condiciones socioeconómica, género, edad, raza o pertenencia étnica, orientación sexual, discapacidad física, entre otros que se convierten en desigualdades u opresiones excluyentes e injustas por una comunidad que no comprende las diferencias. (2017, p.33)

En el año 1990, la Declaración de Jomtien, "Educación para todos”, muestra que sigue la exclusión en el sistema educativo y deja ver la brecha educativa que existe en diferentes países del mundo. Colombia no es la excepción, la política de acceso para cumplir con metas de cobertura se consideró sin que se realizaran modificaciones al sistema educativo, y luego se evidenció que la educación no es pertinente ni es de calidad, porque no responde a las necesidades educativas ni orienta la enseñanza a las formas de aprender de los diferentes estudiantes que estaban accediendo a ella.

Como consecuencia de la Declaración de Jomtien, para esta época la urgencia de la educación estaba en implementar el enfoque de integración centrado en las necesidades educativas especiales de los estudiantes. En otro trabajo sobre lecciones aprendidas del enfoque de integración (Correa, 2014), se concluye que: "la persona objeto de la integración se asume como un beneficiario, quien debe recibir lo que le es ofrecido: una alternativa, pensada como la mejor. No se interroga por la decisión del otro, en tanto se sabe que lo ofrecido es lo mejor" (2014, p. 136). Más adelante, en la reflexión del proceso de integración, se procedió a implementar modelos flexibles en tiempo, metodología y ritmo de aprendizaje, para personas en extraedad.

La Declaración, además, aportó a la promulgación de políticas públicas para cada problemática social, reflejadas en la situación de la discapacidad, las condiciones de equidad de género y de diversidad sexual, culturales (en el caso de las etnias) y ciclos vitales como niñez, juventud, adulto mayor, etc. Esto acarreó un fraccionamiento del ser humano para atender a la situación en particular vulnerada, y no a su integralidad; además promocionó perspectivas educativas, como la integración escolar, para fundamentar que la educación debe responder al principio de desarrollo humano, que requiere apoyo para los estudiantes y debe darse en el contexto cercano a la persona. Esto trajo como consecuencia la implementación de diferentes estrategias en la educación, como los sistemas de apoyo para los estudiantes con discapacidad y la promoción de la etnoeducación para indígenas y afrocolombianos, y posibilitó el ingreso de otros profesionales a la escuela como psicólogos, educadores especiales y etnoeducadores. Así, las poblaciones que estaban en el anonimato empiezan a transitar por el sistema educativo.

La educación inclusiva surge para garantizar el derecho a la educación. Parecía que el derecho a la educación estaba resuelto con las políticas de acceso, por lo que la discusión se trasladó a la permanencia en el sistema y, por ende, la nueva reflexión empezó en la calidad de la educación. Se llegó a plantear que los nuevos sujetos en la educación entorpecían la calidad educativa, y fue necesario asumir la idea que las barreras no eran de los sujetos sino que se encontraban en las actitudes, el currículo y la organización escolar. Por lo tanto, la Unesco definió el derecho a una educación 
de calidad y pertinente "como la cultura de hacer efectivo para todos los niños, niñas, jóvenes y adultos el derecho a la educación, la participación y la igualdad de oportunidades, prestando especial atención a aquellos que viven en situación de vulnerabilidad" (2008, p. 7). La postura va más de allá de un cupo escolar y la escuela debía garantizar los espacios de participación y favorecer desarrollo autónomo en los seres humanos, independiente de sus características de raza, cultura, sexo, idioma, religión, ideología política y procedencia socioeconómica.

Una de las implicaciones de esta propuesta, ha estado en la flexibilidad, para Correa, lleva inherente el abanico de posibilidades desde el currículo, piensa en el aprendizaje de todos los estudiantes, prepara el contexto, se diversifica cuando es necesario y el maestro está en disposición y formado para atender la necesidad educativa cuando se manifiesta, agregando que su orientación es una responsabilidad de la comunidad educativa. (2010, p. 72-73)

La puesta en marcha en Colombia en el año 2006 del programa de educación inclusiva con calidad, por el Ministerio de Educación Nacional en asocio con el Tecnológico de Antioquia, dio un paso importante para el reconocimiento de los invisibilizados haciendo énfasis en la necesidad de actuar en los contextos y no en particularizar en las personas; Correa y Bedoya (2008), investigadores del Tecnológico, proponen el programa como un proceso en tres etapas:

- Contextualización del programa a las condiciones y las características de la diversidad de la población, analizando las barreras y los facilitadores de contexto. Para esto elabora su propio índice de inclusión articulado a la gestión escolar e implementando un plan de actualización docente en el ámbito nacional para acercar a las comunidades educativas al nuevo enfoque.

- Estabilización mediante el desarrollo de planes de mejoramiento que, caracterizados en el enfoque de inclusión, impulsarán la innovación educativa, especialmente en las formas de enseñar y evaluar y en la corresponsabilidad. La institución es un sistema, y como tal su intervención debe darse con el apoyo de diferentes sectores para atender las desigualdades sociales existentes.

- Profundización, orientada a consolidar las políticas educativas en inclusión, transformar los sistemas de gestión escolar de las instituciones educativas, visibilizar buenas prácticas en inclusión, evaluar los resultados y el impacto de la inclusión en cada institución e instaurar la política del mejoramiento continuo.

La implementación de este programa visibiliza la situación de vulnerabilidad de otros grupos que estaban invisibilizados en el sistema, como la población rural dispersa, las víctimas del conflicto armado, los desplazados y la población de frontera; se promocionan didácticas y modelos flexibles en tiempos y alcance en grados escolares, caracterizados por estrategias organizativas del currículo y tiempos diferenciales, de acuerdo con las necesidades de aprendizaje de los diferentes estudiantes. 
La implementación de la inclusión como enfoque de la educación evidencia que se debía diferenciar en dos grandes conceptos, ambos presentes en la realidad: diversidad como una condición humana y vulnerabilidad como una situación social. Los aportes de la metodología de la interseccionalidad llevan a reflexionar sobre la fragmentación que han tenido las políticas públicas, centradas en condiciones y situaciones particulares del ser humano, aun en el debate nacional sobre el cuál debe ser la estructura de dichas políticas. Por ejemplo, una mujer puede presentar una discapacidad intelectual, pertenecer a una comunidad afrocolombiana y hallarse en una situación de desplazamiento.

Las décadas de 1990-2010, se caracterizan porque la educación se enfocó en compensar las carencias educativas de los diferentes grupos minoritarios en el país, se definieron políticas para el respeto a la primera lengua, en el caso de los indígenas y las personas sordas, para la educación bilingüe para sordos, afrocolombianos, población de frontera y los raizales (población de la isla de San Andrés), para la flexibilidad curricular para estudiantes con discapacidad, población rural dispersa y adultos, así como propuestas pertinentes para víctimas del conflicto armado. En este proceso el Ministerio de Educación ha aportado guías y orientaciones educativas para apoyar la enseñanza y el aprendizaje de diferentes tipos de estudiantes.

Desde el año 2010, el Ministerio de Educación pareciera dar por hecho que estas propuestas se implementan en el país en la responsabilidad que tienen las secretarías de educación de los departamentos y municipios, de igual manera ha promulgado las orientaciones para los estudiantes con discapacidad (2012 y 2017), las orientaciones de educación superior inclusiva (2013) y las orientaciones para la atención educativa de estudiantes con talentos (2015).

En su alcance, estas orientaciones establecen que la educación inclusiva está relacionada con la capacidad de potenciar y valorar la diversidad (entendiendo y protegiendo las particularidades), promover el respeto a ser diferente y garantizar la participación de la comunidad dentro de una estructura intercultural en los procesos educativos, articular los diferentes niveles de la educación y revisar las barreras para el aprendizaje y la participación propia del sistema; además, promocionan el enfoque diferencial, definido como un método de análisis y de acción que hace visibles las formas de exclusión de la educación superior de los estudiantes de los grupos mencionados, para brindar adecuada protección en el sistema educativo.

En la última década, la educación ha tenido que enfrentar una tensión permanente con la globalización de la economía, los ránkings y las evaluaciones externas presionan por una educación más universal y los saberes son cada vez más parecidos entre los países. Esta situación pone en desventaja a los grupos minoritarios que ingresan al sistema con otras visiones del mundo, culturas, prácticas y saberes, y trae como consecuencia la agudización de las brechas educativas. 
Hasta aquí se evidencia que ha prevalecido la formulación de políticas y estrategias organizativas en la escuela y la gestión de procesos por encima de la atención a las necesidades reales de aprendizaje de los estudiantes, se multiplican los criterios para fraccionar al ser humano y la categoría del otro excluido es más compleja de entender en cada sector de la sociedad y en la educación. En este sentido, Gentili expresa: "los que están excluidos del derecho a la educación no lo están solo por permanecer fuera de la escuela, sino también por ser parte de un conjunto de relaciones y circunstancias que los alejan de este derecho" (2011, p. 80). El derecho a la educación no se logra con la superación parcial de las condiciones que la han negado en el pasado, implica la superación efectiva de tales condiciones y la sostenibilidad en el tiempo con acciones preventivas en la exclusión.

En los últimos años, abundan las políticas educativas para garantizar el derecho a la educación de grupos reconocidos como minoritarios, por la existencia y persistencia de desigualdades educativas. Las recomendaciones se han tratado en el campo de la educación superior y se han puesto en marcha frente a la invisibilización de sus prácticas sociales, porque los currículos de los programas académicos siguen siendo hegemónicos, desconocen la realidad de los sujetos, y en la tendencia actual de la justicia educativa y social se reclama que "la institución escolar desempeña un papel fundamental también en la conquista de sociedades más justas y democráticas" (Torres, 2011, p. 266).

Santomé Torres plantea que los movimientos sociales han brindado posibilidades para establecer nuevas concepciones en las prácticas sociales, y referencia el caso de la "dignidad humana" a partir del movimiento LGBT, el cual ocurre paralelo al de "la sostenibilidad de la vida en la tierra"; este último es incluso objeto de interés de diferentes disciplinas. Santos, con las epistemologías del sur, reconoce que hoy existe la urgencia por actuar, que se debe fundamentar en un pensamiento alternativo que, en la "inagotable diversidad humana", considere alternativas "culturales, políticas, sociales y económicas” y supere el "pensamiento ortopédico" centrado en la táctica y la reforma para mantener el poderío sobre la humanidad, y convoque al reconocimiento de la presencia de dos tendencias que afectan a la humanidad, una en la sostenibilidad y otra en las condiciones y dinámicas de las personas en sus contextos, y así avanzar de la dinámica de "movimientos sociales" particulares a los grupos, a la de “intermovimientos sociales". Se reitera la necesidad de un "pensamiento crítico social y político” y se apela al cambio en las respuestas, las cuales tendrán que centrarse en “posiciones estratégicas y revolucionarias”. En esta línea, Rosana Sosa expresa:

Se hace necesario subrayar que la universidad, en este contexto, debe aspirar a una reformulación no solo del modo en que se ocupa de sus quehaceres tradicionales — la formación y la producción de conocimiento— sino del modo en que ha de ocupar el espacio público. En este marco, pensar una apertura hacia "otros saberes", producidos por "otros actores", siguiendo "otras lógicas" pareciera configurar un intersticio interesante en el que la universidad ha de construir y liderar el espacio en el que se propicie y se promueva la articulación de los saberes social e históricamente producidos. (2011, p. 206) 
La institución que se reafirma en una educación orientada hacia la diversidad poblacional, en su posicionamiento tiene dos miradas, exógena y endógena. La primera da cuenta de factores concomitantes a la educación que están relacionados con las necesidades básicas del ser humano y que pueden estar interfiriendo con el acceso a la educación, su intervención es intersectorial; la segunda se relaciona con la participación en la vida escolar, con experiencias pertinentes para el acceso al aprendizaje, y con los momentos de promoción de grado escolary de transición entrelos niveles de la educación. Ambas miradas son importantes para realizar construcciones educativas que impacten en el contexto social. Al centrarnos en las endógenas, es necesario revisar la dinámica institucional en la dimensión de lo instituyente, que la define Fernández como "el cuestionamiento, la crítica, y la propuesta opuesta o de transformación”, y propone "observar desde lo más circunscrito hacia lo más amplio" (1993, pp. 36-37), y allí a "las organizaciones en donde se incluyen los grupos y los procesos de ayuda, dirección y control que originan", así como "la estructura y funcionamiento de las matrices grupales en donde se insertan esas relaciones interpersonales y las formas como influyen sobre ellas".

Las epistemologías del sur aportan en estos vacíos de saberes humanistas de los grupos en la educación superior y proponen la decolonialidad del conocimiento para darles apertura a saberes que han sido inferiorizados producto de la colonización y que se reconocen como pérdida de una autorreferencia genuina de las prácticas sociales. Santos da a conocer que

Cuando estudiantes de grupos minoritarios entran a la universidad y verifican que su inclusión es una forma de exclusión: se enfrentan con la tabla rasa que está hecha a partir de sus culturas y de los conocimientos propios de las comunidades de donde son originarios. (2007, p. 45)

\section{Conclusión}

Lo expuesto deja ver que han existido políticas y conceptualizaciones para aportar a la educación de grupos excluidos y discriminados, así se promuevan nuevos paradigmas para la atención educativa, los cuales se espera que marquen nuevos horizontes, que surjan dentro de la escuela y se sitúen libres de prejuicios con relación al aprendizaje del ser humano, además, que le den la voz a los grupos reconocidos como minoritarios para participar en dichas construcciones, incluido el currículo del programa académico al que ingresan en la educación superior. 


\section{Referencias}

Abrahamson, P. (1997). Exclusión social en Europa: ¿vino viejo en odres nuevos? en L. Moreno (comp.), Unión Europea y Estado del Bienestar (pp. 117-141). Madrid: CSIC

Correa, J. (2018). Tesis doctoral "La construcción de la justicia curricular en la educación superior, situada en la ecología de saberes de grupos minoritarios discriminados y excluidos”. Doctorado en Humanidades de la Universidad Nacional de Rosario Argentina.

Correa, J, y Restrepo, N. (2017). Modelo para la atención a la diversidad en el Tecnológico de Antioquia centrado en la justicia educativa y la equiparación de oportunidades. Medellín: Publicar T.

Correa, J., Bedoya, M. y Agudelo, G. (2015). Formación de docentes participantes en el programa de educación inclusiva con calidad en Colombia. Revista Latinoamericana de Educación Inclusiva, 9 (1), 43-61.

Correa, J. (2014). Lecciones aprendidas del enfoque de integración, en Marco conceptual y experiencias de la educación especial en México. Durango: Instituto Universitario Anglo Español.

Correa, J. (2010). Evaluación psicopedagógica en el contexto de atención a la diversidad. Revista Senderos Pedagógicos. (1), 67-74.

Correa, J. y Bedoya, M. (2008). Guía y Herramienta Educación inclusiva con calidad: "construyendo capacidad institucional para la atención a la diversidad". Medellín: Divegráficas.

Dussel, E. (1997). Ética de la liberación en la edad de la globalización y de la exclusión. Madrid: Trotta.

Echavarria, D., Urrea, D. y Zapata, A. (2015). La diversidad se debe hablar distinto. Lenguajes discriminatorios y antidiscriminatorios en las narrativas discursivas de niñas y niños. Revista Senderos Pedagógicos. (6), 53-67.

Fernández, L. (1993). Instituciones educativas Dinámicas institucionales en situación de críticas. Buenos Aires: Paidós.

Gentili, P. (2015). Pedagogía de la igualdad. Buenos Aires: Siglo Veintiuno.

Henderson, J. (2006). La modernización en Colombia: los años de Laureano Gómez, 1889-1965. Colección Clio. Medellín: Editorial Universidad de Antioquia, pp. 415-416.

Santos, B. (2005). Renovar la teoría crítica y reinventar la emancipación social. Buenos Aires: CLACSO.

Sosa, R. (2011). Los saberes "necesarios": la producción y legitimación social. El rol de la universidad. Científicas Argentinas del CONICET, XV (17), 2001-2011.

Torres, S. (2011). La justicia curricular: el caballo de Troya de la cultura curricular. España: Morata.

UNESCO. (2007). Conferencia internacional de educación. La educación inclusiva: el camino hacia el futuro. Ginebra 25 a 28 de noviembre de 2008.

UNESCO. (1948). Declaración Universal de Derechos Humanos. 